\title{
The use of hysteroscopy in endometrial cancer: old questions and novel challenge
}

\author{
Péter Török, Szabolcs Molnár, Rudolf Lampé, Attila Jakab
}

Department of Obstetrics and Gynecology, Faculty of Medicine. University of Debrecen. Hungary

Szabolcs Molnár: szmolnar.md@gmail.com

Rudolf Lampé: lampe.rudolf@med.unideb.hu

Attila Jakab: ja@med.unideb.hu

\section{Corresponding author:}

Péter Török MD, PhD

Department of Obstetrics and Gynecology

University of Debrecen. Faculty of Medicine.

petertorokdr@gmail.com

$+36703158024$

Nagyerdei krt 98, 4032 Debrecen - Hungary

Keywords: endometrial cancer, hysteroscopy, MRI, curettage, ultrasound, staging

Conflict of interest: The authors report no conflict of interest. 


\begin{abstract}
Endometrial cancer is the most common gynecological malignancy with relatively good overall prognosis. It has traditionally two subtypes: Type 1 (endometrioid carcinoma) and type 2 (non-endometrioid). The prognosis is excellent of Stage-I endometrioid cancer, with a 5-year survival rate of $96 \%$. However, the prognosis is much worse for women with high-risk endometrial cancer. Effective preoperative staging is important, in order to tailor treatment and achieve optimal long-term survival. The majority of asymptomatic polyps detected by ultrasound and are treated surgically. Conventionally, D\&C were performed to get a histological diagnosis, but nowadays the hysteroscopy with biopsy starts to be considered as the gold standard. Hysteroscopic resection seems to reduce the risk of under-diagnosed (atypical endometrial hyperplasia) endometrial cancer. To avoid spreading of malignant cells, hysteroscopy should be performed with concern in keeping low intrauterine pressure. In comparison with cervical injection, the hysteroscopic way had a better detection rate in the para-aortic area during sentinel lymph-node mapping. In the assessment of cervical involvement the accuracy of MRI was significantly higher than the accuracy of HSC. In fertility sparing cases hysteroscopic endometrium resection with progesterone therapy is an acceptable option.
\end{abstract}




\section{Introduction}

Endometrial cancer is the most common gynecological malignancy with relatively good overall prognosis [1].Nearly 320000 new cases are diagnosed in every year [1]. The incidence of this malignancy is higher in high-income countries (5.9\%) compared with low-resource countries $(4.0 \%)$, but due to the earlier diagnosis and effective treatment the specific mortality is contrary $[1,2]$. The incidence of endometrial cancer is less than two per 100000 females in Middle and Western Africa and more than 16 per 100000 in North America [2]. The overall survival (OS) has improved in the last half-century from $63 \%$ to $80 \%$, the 5-year OS is highly dependent from stage of the disease, it varies from $90 \%$ in stage I, to $20 \%$ in stage IV [2]. In Europe, the mortality of the disease is the eighth among cancer death in women (2012's data) [1]. Endometrial cancer arises from the epithelial layer of the uterine cavity $[1,3]$. It has traditionally two subtypes: Type 1 (grade 1 and 2 endometrioid carcinomas) are the most common endometrial cancers [1]. They are linked to excess and unopposed of estrogen stimulation and can originate from atypical endometrial hyperplasia [1]. Type 1 cancers are usually diagnosed at early stages and have a good prognosis [1, 4]. Type 2 are rare conditions including grade 3 endometrioid cancers as well as non-endometrioid, which are diagnosed typically in advanced stages and have a poor prognosis $[1,5,2]$. The prognosis is excellent of Stage-I endometrioid cancer, with a 5-year survival rate of $96 \%$. However, the prognosis is much worse for women with high-risk endometrial cancer (Grade 3 or non-endometrioid histotype and/or Stage $\geq \mathrm{IB}$ ), because these women are at increased risk of lymph node metastasis, distant tumor spread and tumor recurrence [6]. Thus, effective identification of high-risk patients preoperatively is important, in order to tailor treatment and achieve optimal long-term survival.

Exceptionally among gynecological malignancies, the endometrial cancer has usually an early sign, the abnormal uterine bleeding (AUB). AUB can indicate endometrial cancer more often, when it occurs in the postmenopausal age. The postmenopausal bleeding is a frequent condition that can affect $7-15 \%$ of women in postmenopausal age. Prevalence of malignant endometrial disease is wide in these population, it varies from $3 \%$ to $10 \%$ [7]. Nevertheless, not all women with endometrial cancer have bleeding [2]. During the evaluation of AUB the first step is transvaginal ultrasound (TVU) examination of endometrial thickness [3]. According to most guidelines, every postmenopausal patients with an increased endometrial 
thickness $(>5 \mathrm{~mm})$, with or without AUB, should undergo to further investigation [3]. The prevalence of endometrial cancer is $<1 \%$ in cases of regular endometrium, with a thickness of less than $4 \mathrm{~mm}[7]$.

In our literature review, no age-specific contraindication or restriction was encountered in the use of hysteroscopy. If the procedure is performed under general anesthesia, the general status of the patient should be suitable for the surgery [8]. In patients with serious medical conditions such as hemodynamic instability, laryngospasm, bronchial asthma, and a previous reaction to general anesthesia, the rate of failure of anesthesia is higher [9]. Hysteroscopy performed in an outpatient setting allows this technique for these patients, too. Fluid overload should be considered as rare, but important complication. Volume overload may cause pulmonary edema and congestive heart failure. Water intoxication may lead to hyponatremia, hypoosmolarity, and cerebral edema [10]. In elder ages these conditions can occur more frequently. Using of inhaled sevoflurane instead of intravenous propofol during operative hysteroscopy results significantly increased glycine $1.5 \%$ absorption, which can decrease the incidence of TURP syndrome [11]. In conclusion the accurate fluid balancing, limiting the operation time may prevent such complications, which is more important in elder ages [12].

Conventionally, dilatation and curettage $(\mathrm{D} \& \mathrm{C})$ were performed to get a histological diagnosis, but nowadays the hysteroscopy with biopsy starts to be considered as the gold standard because it allows both to visualize all uterine walls and to perform targeted biopsy from localized lesions 


\section{Diagnosis}

In postmenopausal age, endometrial polyps stand commonly behind endometrial thickness [13]. Incidental finding of polyps are common and with the common use and the high imaging quality the transvaginal ultrasound they are detected more often: $44.4 \%$ of the premenopausal polyps and in the $36.1 \%$ of the menopausal polyps were asymptomatic $[14,15,16]$. The majority of asymptomatic polyps detected by ultrasound and are treated surgically, although little is known about their natural behavior. They carry low risk of malignancy, hyperplasia or endometrial cancer in the polyp was found in $1.7 \%$ of premenopausal and in $5.4 \%$ of postmenopausal polyps [17]. Recently, analyzing the natural history by following 112 asymptomatic polyps treated expectantly (6-135 months, median 22.5 ) it was found, that $6.3 \%$ of polyps completely regressed and abnormal bleeding appeared in $15 \%$ independently of the growing pattern. Since that the regression rate is low and cannot be predicted, thus the consideration of surgical treatment of polyp is a reasonable choice, especially in menopause when polyps have higher risk of malignancy. Expert ultrasound scan may add some help to distinguish between polyps with low and high risk of malignancy. Benign polyps characteristically have smooth surface, well-defined hyperechoic borders ("bright edge"), single pedicle artery and morphologically normal endometrium adjacent to the polyp [18]. In contrast, polyps with malignant potential mostly have irregular surface, multiple feeding vessels, abnormal adjacent endometrium and occasionally signs of myometrial invasion (irregular endomyometrial border) [19]. Intensive studies on the detailed ultrasound features of histologically different intracavitary lesions using the features recommended by the International Endometrial Tumour Analysis (IETA) group are still ongoing [1, 20]. While it still remains controversial removing of endometrial polyps in asymptomatic patients, one should keep in mind that only histological evaluation could exclude focal neoplastic lesions. Hysteroscopic removal is simple and effective way of treatment of polyps and secures the patient from the stress and uncertainty of several follow-up examinations. There is little question, that when additional risk factors are present and chronic hyperestrogenic condition on the endometrium clinically can be evaluated (eg. obesity, chronic anovulation, diabetes, tamoxifen treatment, etc.), the intervention is absolutely indicated [13]. 


\section{Accuracy of hysteroscopy}

The use of hysteroscopy (HSC) and the targeted biopsy can help to get proper diagnosis and to reduce the amount of under-diagnosed endometrial cancer. In a recent study the mean risk of finding endometrial cancer at hysterectomy after atypical endometrial hyperplasia diagnosed by uterine curettage was $32.7 \%$, with a risk of $45.3 \%$ after hysteroscopically guided biopsy and 5.8\% after hysteroscopic resection. This rate of under-diagnosed endometrial cancer results in inappropriate surgical procedures $(31.7 \%$ of tubal conservation in data available and no abdominal exploration in $24.6 \%$ of the cases). Hysteroscopic resection seems to reduce the risk of under-diagnosed endometrial cancer [21].

The HSC can be useful in the differential diagnosis of malignant and non-malignant lesions of endometrium. Some research group tried to find special signs and to create scoring systems, which allows the stratification of endometrial pathologies even for less experienced operators. In a recent study a "scoring system" was created to assist in the differential diagnosis between non-pathological endometrium, non-atypical hyperplasia, complex atypical hyperplasia, and well-differentiated endometrioid adenocarcinoma (EC) [4]. This system showed a good diagnostic performance, especially in G1 endometrioid adenocarcinomas, and represented an important diagnostic instrument for the diagnosis of atypical endometrial hyperplasia (AEH) and endometrial cancer [4]. The aim of Pivano's study was to estimate the rate of coexisting endometrial carcinoma or AEH residue in patients with AEH diagnosed by total hysteroscopic resection of the endometrium [5]. The risk of endometrial carcinoma after diagnosis of $\mathrm{AEH}$ on total hysteroscopic resection, without suspicious aspect during hysteroscopy, is low but it still exists. Total hysteroscopic resection may be a possible alternative to hysterectomy in patients with AEH who refuse hysterectomy or are at high surgical risk. In these patients, a regular and long-term follow-up monitoring is necessary due to the low risk of associated residual cancer [5]. 


\section{Safety of Hysteroscopy In Endometrial Cancer}

The use of hysteroscopy in already confirmed endometrial cancer is still controversial. Some studies presented the spreading of washing fluid into the abdominal cavity, but its negative effect on prognosis of the disease is not well established. Some studies indicated a high risk for intraperitoneal cancer cell dissemination during hysteroscopy, but others showed that hysteroscopy did not result in cancer-cell spreading into the peritoneal cavity [22].

To address this issue, Polyzos et co-workers performed a systematic review and meta-analysis aiming to estimate the risk of endometrial cancer cell spreading to abdominal cavity and the risk for disease upstaging associated with the hysteroscopic procedure [22]. Their analysis highlighted that hysteroscopy in patients with endometrial cancer increased the risk of cancer cell seeding into the peritoneal cavity and disease upstaging, compared to no hysteroscopy. This risk was higher with isotonic sodium chloride media and depended from inflation pressure [22].

Baker and Adamson [23] as well as Leveque et al. [24] used high pressure liquid and observed that pressure values of $150 \mathrm{mmHg}$ affected positive results of the cytological examination in $37 \%$ cases compared to $1 \%$ positive results when using pressure values below $100 \mathrm{mmHg}$. Applying lower intrauterine pressure $<70 \mathrm{mmHg}$, significantly lower number of endometrial cells were observed in abdominal cavity [23].

The distension media used for endoscopic procedures in the uterine cavity must be strictly controlled for relatively low pressures to prevent the increase in the risk of endometrial cancer intraperitoneal spread [25]. Consequently, large and adequately powered prospective trials with adequate follow-up are needed to clarify whether the observed cancer cell dissemination may jeopardize patients' prognostic outcomes [22]. Until such a trial becomes available, hysteroscopy should be performed with concern in keeping low intrauterine pressure [22]. 


\section{Staging}

The role of hysteroscopy in the preoperative work-up and staging in endometrial cancer is not clearly defined, yet. At present the Magnetic Resonance Imaging (MRI) is the recommended as a preoperative diagnostic method for assessment of the cervical involvement in endometrial adenocarcinoma. MRI is recommended by The International Federation of Gynecology and Obstetrics (FIGO) and National Comprehensive Cancer Network (NCCN) [26]. This study shows that the accuracy of assessing cervical conditions is $93.2 \%$ by MRI, which is significantly higher than that of hysteroscopy $(55.7 \%)(\mathrm{P}=0.007)$. Similarly, the European Society for Medical Oncology - European Society of Gynaecological Oncology - European SocieTy for Radiotherapy and Oncology (ESMO-ESGO-ESTRO) consensus considers MRI as a useful tool to complete informations regarding the myometrial invasion in those institutions where the lymph node dissection (LND) is tailored according to the stratification of patients into low-, intermediate- and high risk groups [6], ultrasound (US) recommendation, IETA study, $2 \mathrm{D}$ vs $3 \mathrm{D}$. In parallel, they also support the use of the preoperative specialized ultrasound for the patients stratifications (Level of Evidence: IV, Strength of recommendation: A). The power of $2 \mathrm{D}$ and $3 \mathrm{D}$ ultrasound imaging in the endometrial cancer staging was investigated recently by a multicenter study, in which 15 expert examiners off line analyzed 2D videos and 3D volumes of 58 endometrial cancer patients. The interrater reliability was better for $2 \mathrm{D}$ than for $3 \mathrm{D}$, both in the detection of deep myometrial ( $550 \%)$ invasion and cervical involvement. So in expert hands 3D Volume Contrast Imaging (3D-VCI) do not add more information to $2 \mathrm{D}$ for endometrial cancer staging and detecting the cervical stromal involvement is more effective than estimating the deep myometrial invasion [27]. The detailed ultrasound characteristics of endometrial cancer according to stage and grade was also examined by the IETA group in a prospective multicenter study. Grayscale and color Doppler features were found to be different in highand low-risk endometrial cancer and they showed association with tumor grade and stage. These data show, that ultrasound has not reached the limits in endometrial cancer staging yet. Being a reproducible, dynamic and non-invasive examination the routine application of transvaginal ultrasound in endometrial cancer is recommended [28].

Beside MRI and ultrasound, the HSC can be useful method in the examination of cervical involvement of disease. Ávila ML et al. in their study tried to determine the usability of HSC 
in evaluation of cervical involvement in endometrial carcinoma [29]. Rate of real positive result was only $56.36 \%$. This was an excessively high rate of false positives. The use of directed cervical biopsy following positive HSC finding did not increased this rate. Their results did not confirm the utility of HSC in evaluation of cervical spreading of tumor. However, they concluded that hysteroscopy is a good diagnostic method in endometrial carcinoma when the tumor is confined to the uterine corpus [29].

Based on the above the HSC is not reliable to asses cervical involvement, but is MRI more accurate? Guocai Xu et al. compared the diagnostic values of MRI and HSC in the assessment of cervical involvement in their study [26]. The accuracy of MRI was significantly higher (93.2\%) than the accuracy of HSC (55.7\%) [26]. The accuracy, specificity, and positive predictive values of MRI were significantly better than those of HSC, while the sensitivity and negative predictive values were similar. Considering this result MRI is better than HSC for cervical assessment [26].

In other research, the HSC was used differently as a tool in the preoperative workup. Hsuan $\mathrm{Su}$ et al. used a novel hysteroscopic pattern of microvascular architecture in uterine endometrioid adenocarcinoma. They hypothesized that a novel specific glomerular pattern might be of value to prognosticate EC. This study aimed to find out the accuracy of this specific pattern identified and described for the first time by us, in risk stratification of endometrial carcinoma [30]. The glomerular pattern was present in a high percent of patients with moderate or poorly differentiated endometrial carcinoma (96\%). But this study was not absolutely representative due to it's single centre, single expert, single-arm nature [30].

No data are available to establish whether operative hysteroscopy is superior to standard Pipelle sampling to determine histological and grade status in endometrial cancer (EC). Phelippeau J et al. evaluated whether Pipelle device sampling or operative hysteroscopy had an impact on preoperative determination of histological and grade status in EC and consequently on surgical management [31]. Discrepancies in the pathological analysis were assessed between Pipelle sampling or operative hysteroscopy and the final histology. By their results both Pipelle device sampling and hysteroscopy showed a limited accuracy in assessing histological type and grade in EC. In cases of $20 \%$, status is underestimated or overestimated by final analysis which would lead to inappropriate initial management [31]. 


\section{Sentinel lymph node mapping}

Surgical staging with lymphadenectomy defines recurrence risk and helps clinicians to decide whether to give adjuvant treatment to high-risk patients [32]. Complete pelvic and para-aortic lymphadenectomy can be associated with major co-morbidities including lymphoedema, lymphocyst formation, and genitofemoral nerve injury, and is technically difficult to achieve in obese populations [32]. The first report according to application of sentinel lymph node (SLN) mapping in endometrial cancer was presented in 2004. There are several ways to target the tracer, one of them is the hysteroscopic injection. Fabio Martinelli et co-workers aimed to present one of the largest series published on hysteroscopic injection of tracers for SLNs mapping in endometrial cancer. They analyzed the detection rate and diagnostic accuracy of SLNs mapping following hysteroscopic injection of tracer. Moreover they compared two tracer materials the Indocyanine Green (ICG) and Tc99m [33, 34]. They found that this method is accurate and has a good detection rate. In comparison with cervical injection, the hysteroscopic way had a better detection rate in the para-aortic area. Moreover the use of ICG assured a better bilateral detection rate [33, 34]. In contrast with Martinelli's result in the FIRES trial cervical injection achieved a higher overall detection frequency for sentinel lymph nodes, with a similar anatomic distribution when compared with endometrial injection [32]. In conclusion the place of HSC in SNL remains still controversial. 


\section{Treatment - Fertility preservation}

Endometrial cancer occurs rarely in the young population and the preservation of fertility can be necessary among these patients. The decision should always be based on stage and grade of disease. Most stage I, grade 1 endometrial cancers are curable with surgical resection and the prognosis is good. A conservative approach to treat this specific group is an important issue. Hysteroscopic tumor resection is an option for early-stage endometrial cancer and additional progestin therapy could be considered as a safe treatment strategy. The pregnancy rate of combined therapy seems not inferior to the hormone therapy alone [35].

Some studies examined the safety of hysteroscopic resection of premalignant and malignant lesions of uterine cavity. Osnat Elyashiv et al. evaluated the residual uterine pathology after hysteroscopic resection of premalignant and malignant endometrial polyps [36]. Their study showed that $91.2 \%$ of women undergoing hysteroscopic endometrial polypectomy had residual lesions in their uteri. Additionally, when the rest of the endometrial lining appeared to be normal on hysteroscopic view, $55.6 \%$ had multifocal disease. Hysterectomy after hysteroscopic polypectomy maybe needed, but the option of progestin treatment after polypectomy can be offered with caution to young patients with intention to start a family. Total hysteroscopic endometrial resection may also be reserved for postmenopausal women who have high surgical risk or who decline hysterectomy [36].

Fangfang Wang et al. focused in their work specially on young women with early stage disease who wished to preserve their fertility [37]. HSC was found to be a safe, reliable, and effective procedure for patients with early EC. Due to their study hysteroscopy is one of the most accurate and reliable methods to diagnose intrauterine lesions, especially for early EC, because of the advantages of performing uterine biopsy under direct vision [37].

Sarah Guillon et al. conducted a meta-analysis and systematic review evaluating the impact of patients' characteristics, management, and methods used in studies reporting remission rates in fertility-sparing management for atypical hyperplasia and endometrial cancer [38]. The meta-analysis included 65 studies and 1604 patients and it concluded that operative hysteroscopy as a sampling method and a higher ratio of infertile patients enrolled was associated with a better remission rate. Overall remission rate was 0.75 [38]. There was no consensus regarding the optimal treatment strategy. Based on their analyses operative 
hysteroscopy should be the preferred endometrial sampling method because of its association with a higher remission rate [38].

Taking account of later obstetrical complications and less invasiveness, evaluation and treatment of uterine abnormalities could be performed with our grasping or dilating the cervix in an out-patient setting, as well [39]. During office hysteroscopy detection and resection of the polyp can be carried out using electrosurgical [40] or mechanical instruments [41. In this way hospitalization and anesthesia could be avoided.

In conclusion, the hysteroscopic endometrium resection with progesterone therapy is an acceptable option with good response rate. The careful selection of the candidates and a thorough counseling about tumor treatment and fertility plan are absolutely necessary before starting the treatment [35]. 


\section{Conclusion}

In conclusion the HSC is an essential tool in the diagnosis, preoperative workup and management of endometrial cancer. The use of the technique in already diagnosed EC is safe, because the overall survival and prognosis was not worse despite the presence of tumor cells in peritoneal cytology, but the HSC should be performed with restrictions. The value during sentinel lymph node biopsy is controversial, an account of the better detection rate with cervical injection of tracer. In this aspect of use, further evaluations are needed. Finally, the management of the EC in young patient with fertility potential is almost unimaginable without HSC. It is highly recommended both in treatment and follow up. Moreover, the HSC can use in outpatient setting, by which is a cost-effective tool, and causing less strain and trauma to the patient. 


\section{Referencies}

1Amant F., Mirza MR, Koskas M, et al.: Cancer of the corpus uteri. FIGO CANCER REPORT 2018 Int J Gynecol Obstet 2018;143: 37-50.

2L. S. G. Ulrich Endometrial cancer, types, prognosis, female hormones and antihormones Climacteric, 14:4, 418-425.

3Trojano G, Damiani GR, Casavola VC et al.: The Role of Hysteroscopy in Evaluating Postmenopausal Asymptomatic Women with Thickened Endometrium Gynecology and Minimally Invasive Therapy 2018;7:6-9.

4Ianieri MM, Staniscia T, Pontrelli G et al.: A New Hysteroscopic Risk Scoring System for Diagnosing Endometrial Hyperplasia and Adenocarcinoma Journal of Minimally Invasive Gynecology 2016;23:712-718.

5Pivano A, Crochet P, Carcopino X. et al. Risk of coexisting endometrial carcinoma in case of atypical endometrial hyperplasia diagnosed on total hysteroscopic resection European Journal of Obstetrics \& Gynecology and Reproductive Biology 2016;203:210-213.

6Colombo N, Creutzberg C, Amant F, et al. ESMO-ESGO-ESTRO Consensus Conference on Endometrial Cancer: diagnosis, treatment and follow-up. ESMO-ESGOESTRO Endometrial Consensus Conference Working Group. Ann Oncol. 2016;27:16-41

7Ghoubara A, Sundar S, Ewies AAA Endometrial pathology in recurrent postmenopausal bleeding: observational study of 385 women, Climacteric, 2018;21: 391-396.

8ACOG TECHNOLOGY ASSESSMENT No 13: Hysteroscopy Obstetrics \& Gynecology, 2018;131:151-156

9Centini G, Calonaci A, Lazzeri L et al: Parenterally Administered Moderate Sedation and Paracervical Block Versus General Anesthesia for Hysteroscopic Polypectomy: A Pilot Study Comparing Postoperative Outcomes J Minim Invasive Gynecol. 2015;22:193-198

10Hsieh MH, Chen TL, Lin YH et al.: Acute pulmonary edema from unrecognized high irrigation pressure in hysteroscopy: a report of two cases J Clin Anesth. 2008;20:614-617 
11Munmany M, Gracia M, Nonell R et al: The use of inhaled sevoflurane during operative hysteroscopy is associated with increased glycine absorption compared to intravenous propofol for maintenance of anesthesia. Journal of Clinical Anesthesia 2016;31:202-207

12Silva Santos AM, Coelho D: Operative Hysteroscopy Intravascular Absorption Syndrome: The Gynaecology’s TURP Syndrome-A Case Report. Anesth Pain Med. 2019;9:e90285.

13Uglietti A, Buggio L, Farella $M$ et al.: The risk of malignancy in uterine polyps: A systematic review and meta-analysis. European Journal of Obstetrics \& Gynecology and Reproductive Biology 2019;237:48-56.

14Hassa H, Tekin B, Senses T, Kaya M, Karatas A. Are the site, diameter, and number of endometrial polyps related with symptomatology? Am J Obstet Gynecol 2006;194(3):718. Visualization of the feeding artery with color-Doppelr improves the detection rate.

15Timmerman D, Verguts J, Konstantinovic ML, Moerman P, Van Schoubroeck D, Deprest J, van Huffel S. The pedicle artery sign based on sonography with color Doppler imaging can replace second-stage tests in women with abnormal vaginal bleeding. Ultrasound Obstet Gynecol 2003;22(2):166-171.

16Jakab A, Ovari L, Juhasz B, Birinyi L, Bacsko Gy, Toth Z. Detection of feeding vessels improves the ultrasound diagnosis of endometrial polyps in asymptomatic patients. Eur $\mathbf{J}$ Obstet Gynecol Reprod Biol 2005; 119: 103-107.

17Lee SC, Kaunitz AM, Sanchez-Ramos L, Rhatigan RM. The oncogenic potential of endometrial polyps: a systematic review and meta-analysis. Obstet Gynecol 2010;116(5):1197.

18 Valentin L. Imaging techniques in the management of abnormal vaginal bleeding in nonpregnant women before and after menopause. Best Pract Res Clin Obstet Gynaecol. 2014;28:637-54.

19Alcazar JL, Castillo G, Minguez JA, et al. Endometrial blood flow map- ping using transvaginal power Dopper sonography in women with post- menopausal bleeding and thickened endometrium. Ultrasound Obstet Gynecol 2003;21:583-588.

20Leone FP, Timmerman D, Bourne T, et al. Terms, definitions and measurements to describe the sonographic features of the endometrium and intrauterine lesions: a consensus 
opinion from the International Endometrial Tumor Analysis (IETA) group. Ultrasound Obstet Gynecol. 2010;35:103-12.

21Bourdel N, Chauvet P, Tognazza E et al. Sampling in Atypical Endometrial Hyperplasia: Which Method Results in the Lowest Underestimation of Endometrial Cancer? A Systematic Review and Meta-analysis. Journal of Minimally Invasive Gynecology 2016;5:692 - 701.

22Polyzos NP, Mauri D, Tsioras S, et al.: Intraperitoneal Dissemination of Endometrial Cancer Cells After Hysteroscopy A Systematic Review and Meta-Analysis Int J Gynecol Cancer 2010;20: 261-267

23Baker VL, Adamson GD. Threshold intrauterine perfusion pressures for intraperitoneal spill during hydrotubation and correlation with tubal adhesive disease. Fertil Steril. 1995; 64:1066-1069,

24Leveque J, Goyat F, Dugast J, et al. Value of peritoneal cytology after hysteroscopy in surgical stage I adenocarcinoma of the endometrium. Oncol Rep. 1998 May-Jun;5(3):713-5.

25Stachowicz N, Mazurek D, Łoziński T, et al. Diagnostic hysteroscopy and the risk of malignant cells intraabdominal spread in women with endometrial cancer Ginekologia Polska 2017; 88: 562-567.

26Guocai Xu et al.: Diagnostic Value of Assessment of Cervical Involvement in Early-Stage Endometrial Adenocarcinoma: Comparison of Magnetic Resonance Imaging (MRI) Versus Hysteroscopy Med Sci Monit, 2018; 24: 7952-7957.

27Green RW, Valentin L, Alcazar J, et al. Endometrial cancer off-line staging using twodimensional transvaginal ultrasound and three-dimensional volume contrast imaging: Intermethod agreement, interrater variability and diagnostic accuracy. Gynecol Oncol 2018; 150: 438-445.

28Epstein E, Fischerova D, Valentin L, et al. Ultrasound characteristics of endometrial cancer as defined by International Endometrial Tumor Analysis (IETA) consensus nomenclature: prospective multicenterstudy. Ultrasound Obstet Gynecol. 2018;51:818-828.

29Ávila ML, Ruiz R, Cortaberria JR, et al. Assessment of cervical involvement in endometrial carcinoma by hysteroscopy and directed biopsy Int $\mathrm{J}$ Gynecol Cancer 2008;18:128-131. 
30Su H, Pandey D, Liu LY et al.: Pattern Recognition to Prognosticate Endometrial Cancer The Science Behind the Art of Office Hysteroscopy - A Retrospective Study Int J Gynecol Cancer 2016;26:705-710.

31Phelippeau J, Canlorbe G, Bendifallah S, et al.: Preoperative diagnosis of tumor grade and type in endometrial cancer by pipelle sampling and hysteroscopy: Results of a French study Surgical Oncology 2016;25: 370-377.

32Rossi EC, Kowalski LD, Scalici J, et al.: A comparison of sentinel lymph node biopsy to lymphadenectomy for endometrial cancer staging (FIRES trial): a multicentre, prospective, cohort study Lancet Oncol 2017;18:384-92.

33Martinelli F,Ditto A, Signorelli M, et al. Sentinel node mapping in endometrial cancer following Hysteroscopic injection of tracers: A single center evaluation over 200 cases Gynecologic Oncology 2017;146:525-530.

34Martinelli F, Ditto A, Bogani G, et al. Laparoscopic Sentinel Node Mapping in Endometrial Cancer After Hysteroscopic Injection of Indocyanine Green Journal of Minimally Invasive Gynecology 2017;24:89-93.

35Yang HC, Liu JC, Liu FS Fertility-preserving treatment of stage IA, well-differentiated endometrial carcinoma in young women with hysteroscopic resection and high-dose progesterone therapy Taiwanese Journal of Obstetrics \& Gynecology 2019;58:90-93.

36Elyashiv O, Sagiv R, Kerner R, et al. Hysterscopic Resection of Premalignant and Malignant Endometrial Polyps: Is it a Safe Alternative to Hysterectomy? Journal of Minimally Invasive Gynecology 2017;24:1200-1203.

37Wang F, Yu A, Xu H et al. Fertility Preserved Hysteroscopic Approach for the Treatment of Stage Ia Endometrioid Carcinoma Int J Gynecol Cancer 2017;27: 1919-1925.

38Guillon S, Popescu N, Phelippeau J et al. A systematic review and meta-analysis of prognostic factors for remission in fertility-sparing management of endometrial atypical hyperplasia and adenocarcinoma Int J Gynecol Obstet 2019;146:277-288.

39Salazar CA, Isaacson KB. Office Operative Hysteroscopy: An Update. J Minim Invasive Gynecol. 2018 Feb;25(2):199-208 
40Bettocchi S, Ceci O, Di Venere R,et al. Advanced operative office hysteroscopy without anaesthesia: analysis of 501 cases treated with a 5 Fr. bipolar electrode. Hum Reprod. 2002; 17:2435-2438.

41Vitale SG. The biopsy snake grasper sec. VITALE: a new tool for office hysteroscopy. J Minim Invasive Gynecol. 2019 Dec 26. pii: S1553-4650(19)31362-7 